\title{
Pharmacological Effects of Marine-Derived Enterococcus faecium EA9 against Acute Lung Injury and Inflammation in Cecal Ligated and Punctured Septic Rats
}

\author{
Hatem M. Abuohashish $\mathbb{D}^{1},{ }^{1}$ Eman H. Zaghloul $\mathbb{D}^{2},{ }^{2}$ Amany S. El Sharkawy $\mathbb{D}^{2}$, \\ Eman M. Abbas $\left(\mathbb{C}^{2},{ }^{2}\right.$ Mohammed M. Ahmed $\mathbb{D}^{3},{ }^{3}$ and Salim S. Al-Rejaie $\mathbb{C}^{3}$ \\ ${ }^{1}$ Department of Biomedical Dental Sciences, College of Dentistry, Imam Abdulrahman Bin Faisal University, \\ Dammam 31441, Saudi Arabia \\ ${ }^{2}$ National Institute of Oceanography and Fisheries (NIOF), Cairo 11516, Egypt \\ ${ }^{3}$ Department of Pharmacology and Toxicology, College of Pharmacy, King Saud University, Riyadh 11451, Saudi Arabia
}

Correspondence should be addressed to Hatem M. Abuohashish; habuohashish@iau.edu.sa

Received 14 May 2021; Revised 12 September 2021; Accepted 16 November 2021; Published 6 December 2021

Academic Editor: Leila Ktari

Copyright (c) 2021 Hatem M. Abuohashish et al. This is an open access article distributed under the Creative Commons Attribution License, which permits unrestricted use, distribution, and reproduction in any medium, provided the original work is properly cited.

\begin{abstract}
Microorganisms obtained from the marine environment may represent a potential therapeutic value for multiple diseases. This study explored the possible protective role of marine-derived potential probiotic Enterococcus faecium EA9 (E. faecium) against pulmonary inflammation and oxidative stress using the cecal ligation and puncture (CLP) model of sepsis in Wistar rats. Animals were pretreated with E. faecium for 10 days before either sham or CLP surgeries. Animals were sacrificed 72 hours following the surgical intervention. The histological architecture of lung tissues was evaluated as indicated by the lung injury score. In addition, the extend of pulmonary edema was determined as wet/dry weight ratio. The inflammatory cytokines were estimated in lung tissues, including tumor necrosis factor-alpha (TNF- $\alpha$ ), interleukin-6 (IL-6), and interleukin-1 beta (IL-1 $\beta$ ) using the enzyme-linked-immunosorbent-assay (ELISA) technique. Moreover, markers for lipid peroxidation such as thiobarbituric acid reaction substances (TBARs), and endogenous antioxidants, including reduced glutathione (GSH) were determined in lung tissues. Finally, the enzymatic activities of antioxidant enzymes such as catalase (CAT), superoxide dismutase (SOD), glutathione peroxidase (GPx), and glutathione reductase (GR) were assayed in the lungs. Pretreatment with E. faecium markedly attenuated CLP-induced lung injury and pulmonary edema. Markers for inflammation, including TNF- $\alpha$, IL-6, and IL- $1 \beta$ were augmented in the lung tissues of CLP animals, while E. faecium ameliorated their augmented levels. E. faecium pretreatment also restored the elevated TBARS levels and the prohibited CAT, SOD, and GPx enzymatic activities in CLP animals. GSH levels were corrected by E. faecium in CLP animals. The inflammatory and lipid peroxidation mediators were positively correlated, while antioxidant enzymatic activities were negatively correlated with CLP-induced lung injury and pulmonary edema. Collectively, marine-derived Enterococcus faecium EA9 might be considered as a prospective therapeutic tool for the management of pulmonary dysfunction associated with sepsis.
\end{abstract}

\section{Introduction}

Sepsis is a life-threatening condition in which the human body responds to infection that injures the internal organs leading to their dysfunction. This condition might progress to septic shock. The prevalence of sepsis is high despite the advances in medical services and intensive care unit settings.
Studies showed that more than 1 million deaths might occur around the world due to sepsis particularly in the neonatal population $[1,2]$. Sepsis also is associated with low-birthweight infants and long-term adverse consequences. Lungs are one of the most affected organs in sepsis. Sepsis might result in acute lung injury and acute respiratory distress (ARDS), which is a common challenge that triggers the 
mortality rate especially in pediatric patients $[3,4]$. Studies demonstrated that mortalities due to acute lung injury/ ARDS are around 26\% in the United States [5]. Experimental sepsis models include the cecal ligation and puncture (CLP) model. This model is extensively used in rodents to mimic the intensive production of inflammatory mediators such as reactive oxygen species (ROS) and cytokines, which altered respiratory function resulting in pulmonary edema and inflammatory exudate accumulation [6].

Probiotics are nonpathological bacteria that live in and colonize the human intestine. They are a useful tool for the host to counteract the detrimental effects of altered or pathological flora. Several randomized controlled trials introduced probiotics for the management of nosocomial infections, including alimentary tract infections as well as respiratory tract infections in adult and pediatric patients [7-9]. In addition, the use of probiotics offers an advantage over conventional antimicrobial interventions as it has lower antimicrobial resistance. In the last two decades, probiotics were introduced as a therapeutic tool in the treatment of sepsis. Clinical evidence demonstrated that probiotics decrease late-onset sepsis incidence in preterm infants [10]. In addition, supplementation of children suffering from severe sepsis with probiotics for 7 days significantly lowered the levels of inflammatory mediators in one randomized controlled trial [11]. The use of probiotics in experimental animals also attenuated sepsis-associated inflammation and oxidative injuries $[12,13]$. Interestingly, the use of probiotics such as Lactobacillus rhamnosus GG and Bifidobacterium longum [14] and Lactobacillus fermentum [15] was able to reverse the pulmonary dysfunction and inflammation using CLP and lipopolysaccharide (LPS) models of sepsis, respectively.

Probiotic strains such as Lactobacilli, Bifidobacteria, and Streptococcus thermophilus are present in several nutritional products like fermented and unfermented milk, yogurts, some juices, and soy beverages [16]. Currently, there are several attempts to develop probiotics from the marine environment as it represents a potential source of novel microorganisms. These microorganisms may produce a vast diversity of secondary metabolites and bioactive molecules, which cannot be acquired from nonaquatic microorganisms [17]. Moreover, marine lactic acid bacteria have potential applications in different industrial and pharmaceutical perspectives along with their significant environmental role in the transformation of organic materials in marine sediments [18]. In this context, this study is aimed at exploring the possible protective role of marine-derived potential probiotic Enterococcus faecium EA9 (E. faecium) against lung dysfunction and inflammation in the CLP model of sepsis.

\section{Materials and Methods}

2.1. Animals and Ethical Approval. In this study, twenty-four male Wistar albino rats were employed. Experimental animals were kindly provided from the animal house at the Institute of Graduate Studies and Research, Alexandria University, where all surgical procedures, including euthanasia, were conducted. Rats were maintained under a controlled environment, including the acclimatization and experimen- tal treatment periods. All investigational conditions and surgical procedures were conducted in agreement with the National Institute of Health (NIH) guide for the care and use of laboratory animals " 8 th edition." Moreover, the experimental protocol of the present study was also ethically approved by the Institutional Animal Care and Use Committee (IACUC) at Alexandria University (AU14-210126-3-2).

2.2. Experimental Model and Design. Animals were randomly allocated into four groups as follows: (a) sham animals with no CLP or probiotic treatment (Sham), (b) sham animals with probiotic treatment (Sham + E. faecium), (c) CLP animals with no probiotic treatment (CLP), and (d) CLP animals with probiotic treatment (CLP+E. faecium). The surgical procedure of the CLP model of sepsis was conducted according to Zubrow et al. [19]. In brief, animals were generally anesthetized using a single intraperitoneal injection of ketamine (EIPICO, $10^{\text {th }}$ of Ramadan city, Egypt) and xylazine (Adwia, $10^{\text {th }}$ of Ramadan city, Egypt) mixture (60 and $5 \mathrm{mg} / \mathrm{kg}$, respectively). Then, a median laparotomy incision of $15 \mathrm{~mm}$ long was made, and the cecum was visualized and exposed. The distal section of the isolated ileocecal valve/cecum was ligated using a 4-0 silk thread. The bowel continuity was maintained and not disrupted during the ligation process. After ligation, the ligated portion of the cecum was subjected to multiple ( 2 to 3 ) punctures using an 18-gauge syringe needle. Then, it was squeezed to extrude an insignificant volume of feces to assure the opening at the puncture site. At the end of the surgical procedure, the ligated cecum was returned to the abdominal cavity. Sham animals had the same surgical procedure except for the ligation and puncture steps. All animals were provided sterile saline ( $20 \mathrm{ml} / \mathrm{kg}$ body weight) subcutaneously. Postoperative pain was maintained using intraperitoneal injection of ketorolac $(30 \mathrm{mg} / \mathrm{kg}$ ) every $12 \mathrm{~h}$ (Amriya Pharmaceutical Industries, Amriya, Egypt). The marine isolate Enterococcus faecium EA9 (GenBank: MW218438.1) was isolated from the gut of shrimp samples collected from the Mediterranean Sea, Egypt. It was isolated on MRS Agar (De Man, Rogosa and Sharpe) medium (LabM, UK) and further evaluated for having potential probiotic properties (under processing data). The freeze-dried probiotic was resuspended in $1 \mathrm{M}$ $\mathrm{NaCl}\left(1 \times 10^{7} \mathrm{CFU} / \mathrm{ml}\right)$, and a $250 \mu \mathrm{l}$ was given immediately, by oral gavage for 10 days before the CLP surgeries, to each rat in sham + E. faecium and CLP $+E$. faecium groups. 72 hours after the CLP procedure, animals were euthanized using a high dose of inhaled general anesthesia (Aesica Queenborough Ltd., Kent, UK). Lung samples were collected for analysis. The lower lobe of the right lung was used in pulmonary edema estimation, while the remaining section of the right lung was preserved in $10 \%$ formalin solution for histological examination. The left lung samples were stored in $-20^{\circ} \mathrm{C}$ for biochemical analysis.

2.3. Estimation of Pulmonary Edema. The extent of pulmonary edema was determined in the extracted lung tissues by measuring wet/dry. For each animal, the lower lobe of the right lung was isolated and weighed. This was considered the 

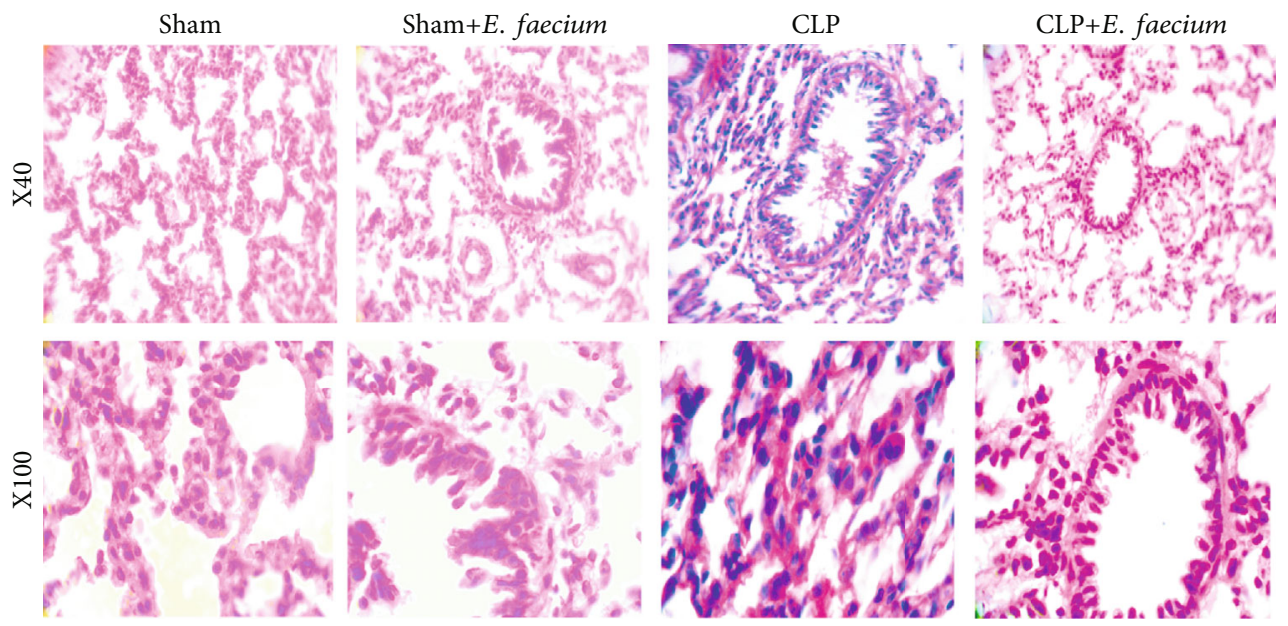

Figure 1: Histological evaluation of marine E. faecium effects on pulmonary inflammation and injury in Wistar rats with sepsis. Images are represented in $\times 40$ and $\times 100$ magnifications. The sham group showed regular lung architecture with no inflammatory exudates and normal hyaline membranes and septal thickening. Similarly, the sham + E. faecium group demonstrated ordinary histological features of lung tissues without inflammation or altered hyaline membranes and septal thickening. In the CLP group, there was a significant inflammatory infiltration of neutrophils and hyaline membranes with marked thickening of the septum. The CLP+E. faecium group revealed low extent of neutrophilic inflammatory infiltrate with mild septal thickening and hyaline deposition.

wet weight. Then, the lobes were dried at $60^{\circ} \mathrm{C}$ for 24 hours. The dried lobes were reweighed to calculate the wet/dry ratio.

2.4. Histological Examination. The right lung of each rat was fixed in a $10 \%$ formalin solution (Sigma-Aldrich, MO, USA). Samples were embedded in paraffin blocks and sectioned using a microtome. Afterward, the sectioned samples were stained with hematoxylin and eosin (Sigma-Aldrich, MO, USA). Slides were examined under a microscope (Leica Biosystems Melbourne Pty Ltd., Melbourne, Australia) and scored blindly as previously described [20]. Lung injury was given a score out of 100 based on the occurrence of inflammatory infiltration from neutrophils, structure of the hyaline membrane, airspaces, and septal thickening.

2.5. Detection of Pulmonary Oxidative Stress Biomarker. The left lung samples were homogenized in a physiological buffer $(1: 10, w / v)$. Thiobarbituric acid reactive substance (TBARS) level was determined, as a marker for lipid peroxidation, while reduced glutathione (GSH) level was measured, as a marker for endogenous antioxidant, using commercially diagnostic kits (Cayman Chemical Co., USA). The homogenate was then centrifuged to produce the postmitochondrial supernatants. Enzymatic activities of antioxidant enzymes such as catalase (CAT), superoxide dismutase (SOD), glutathione reeducates (GR), and glutathione peroxidase (GPx) were estimated by assay kits (R\&D Systems Inc., USA).

2.6. Detection of Pulmonary Inflammatory Cytokines. In the left lung tissue homogenates, the levels of inflammatory cytokines, including tumor necrosis factor-alpha (TNF- $\alpha$ ), interleukin-6 (IL-6), and interleukin-1 beta (IL-1 $\beta$ ) were quantitatively determined using the enzyme-linked-immunosorbent-assay (ELISA) technique following instructions provided by the kits (R\&D Systems Inc., USA).
2.7. Statistical Analysis. The numerical values of the results were presented in the form of the mean of each group \pm standard deviation of the mean (SD). One-way analysis of variance (ANOVA) was used as a statistical analysis test to evaluate the difference between the four groups. One-way ANOVA was followed by the Student-Newman-Keuls post hoc test. Data were analyzed using GraphPad Prism 5 (GraphPad Software, Inc., La Jolla, CA, USA). The difference between the means was considered significant when $p$ values were equal to or less than 0.05 .

\section{Results}

Results of the histological investigation in lung tissues showed that animals in the sham group have a normal appearance and histological features. There were no signs of inflammatory infiltration or thickening of the septum. Sham animals with E. faecium pretreatment also had normal lung histology without inflammation or thickening as compared to sham animals. However, animals in the CLP group had marked inflammation infiltration and altered hyaline membrane. Pretreatment of CLP animals with marine-derived E. faecium markedly restored the histological characteristics of lung tissues, lowered the neutrophilic inflammatory infiltration, and attenuated septal thickening and deposition of the hyaline membrane (Figure 1).

Quantitative scoring of the histological slides revealed a significant $(p \leq 0.05)$ increase in the lung injury score in the CLP group as compared with the sham group. When comparing the CLP group with sham and CLP animals with E. faecium pretreatment, the CLP group showed a significant $(p \leq 0.05)$ higher lung injury score as compared to E. faecium-pretreated groups (Figure 2(a)). Pulmonary edema was assessed as a wet/dry ratio of lung tissues. The CLP group had a significant ( $p \leq 0.05)$ high wet/dry ratio as compared to sham animals. Moreover, the wet/dry ratio was 


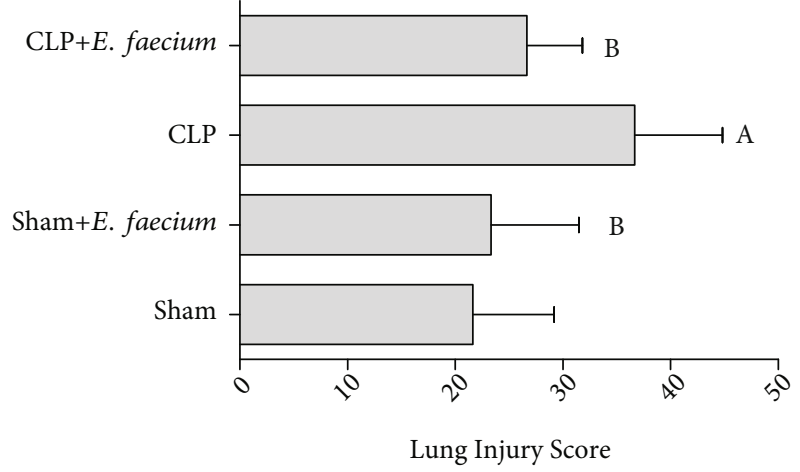

(a)

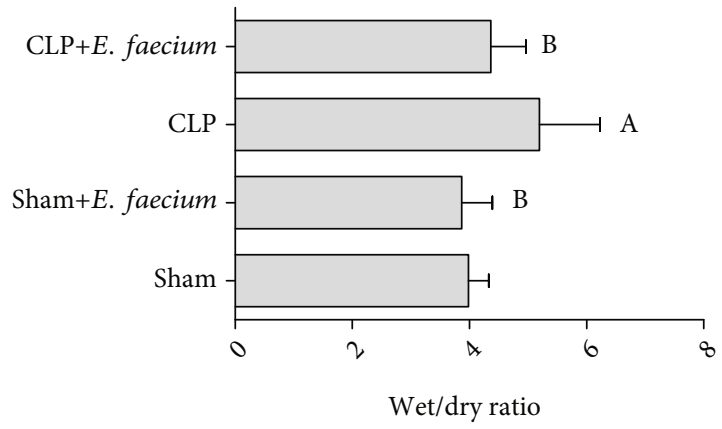

(b)

FIGURE 2: Effects of marine E. faecium pretreatment in Wistar rats with sepsis on (a) lung injury score determined by histological analysis and (b) pulmonary edema expressed as wet/dry ratio. Data are presented as mean $\pm \mathrm{SD}(n=6)$ and statistically analyzed by one-way ANOVA followed by the Student-Newman-Keuls post hoc test. Statistical significance was considered when $p \leq 0.05$. Statistical significance was considered when "A" vs. sham group and "B" vs. CLP group.

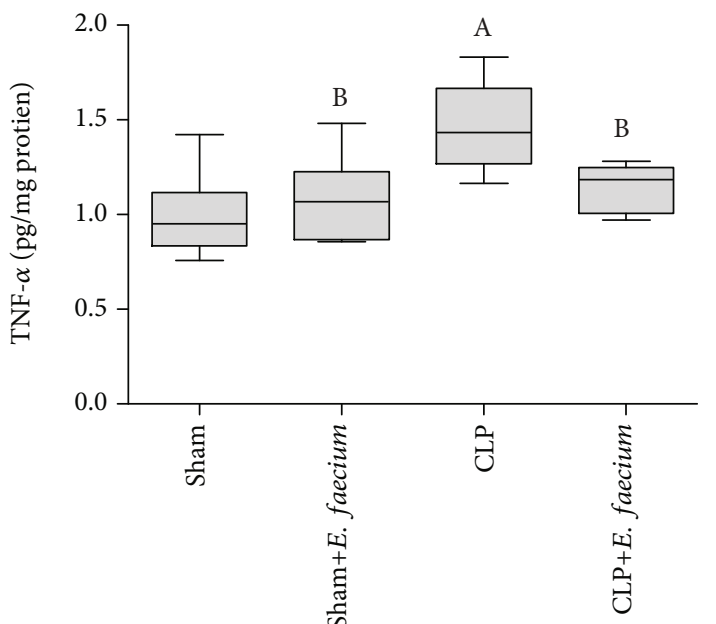

(a)

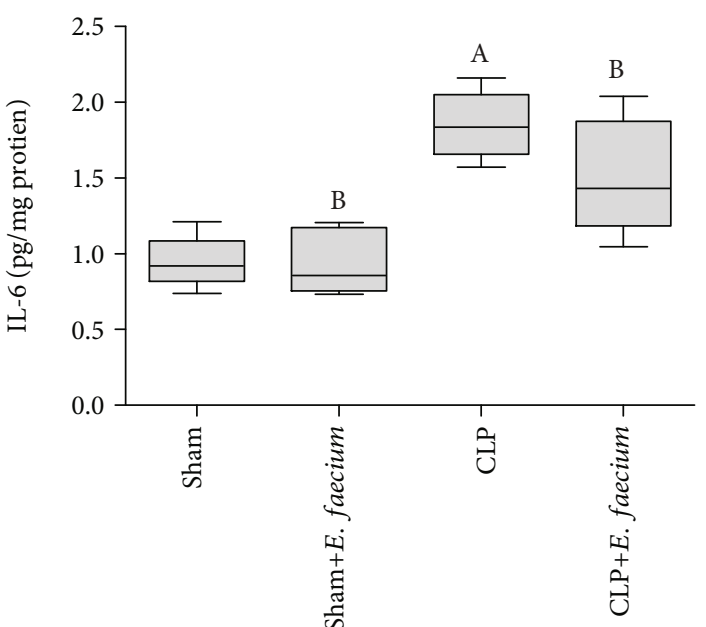

(b)

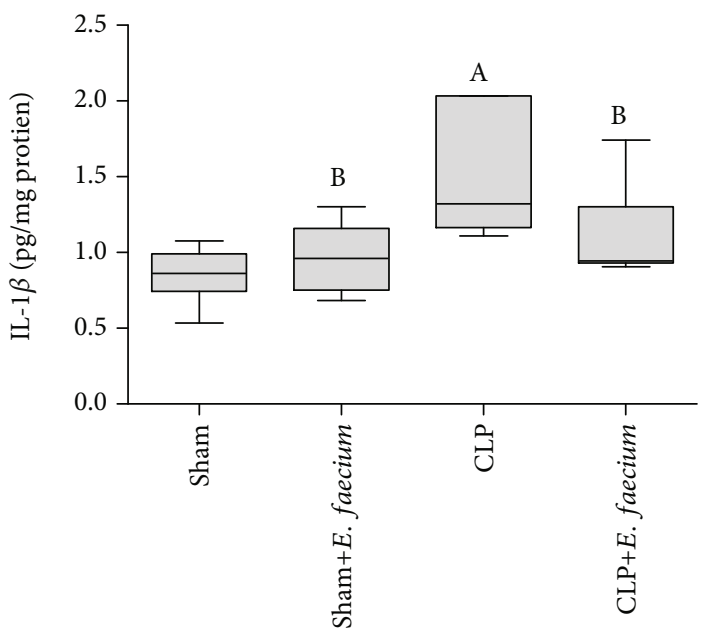

(c)

FIGURE 3: Effects of marine E. faecium pretreatment in Wistar rats with sepsis on lung levels of (a) tumor necrosis factor-alpha (TNF- $\alpha$ ), (b) interleukin-6 (IL-6), and (c) interleukin-1 beta (IL-1 $\beta)$. Data are presented as mean \pm SD $(n=6)$ and statistically analyzed by one-way ANOVA followed by the Student-Newman-Keuls post hoc test. Statistical significance was considered when $p \leq 0.05$. Statistical significance was considered when "A" vs. sham group and "B" vs. CLP group. 


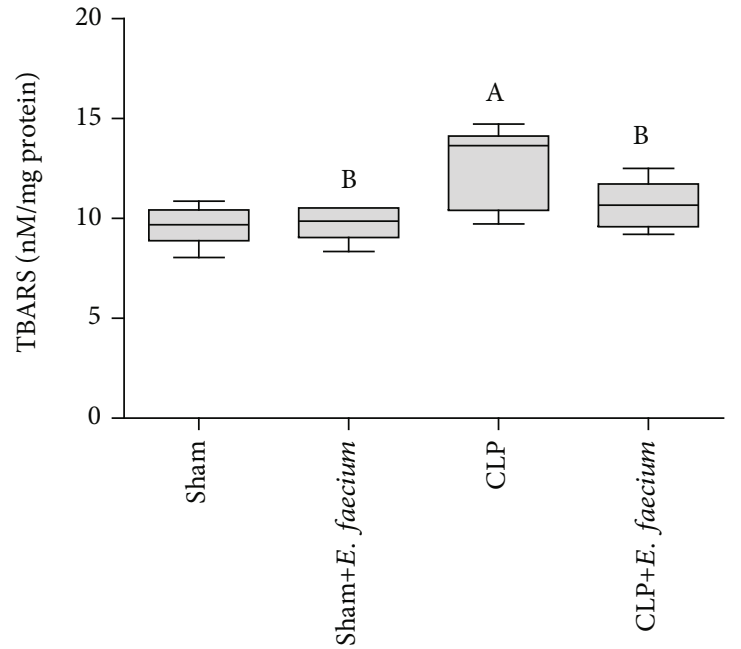

(a)

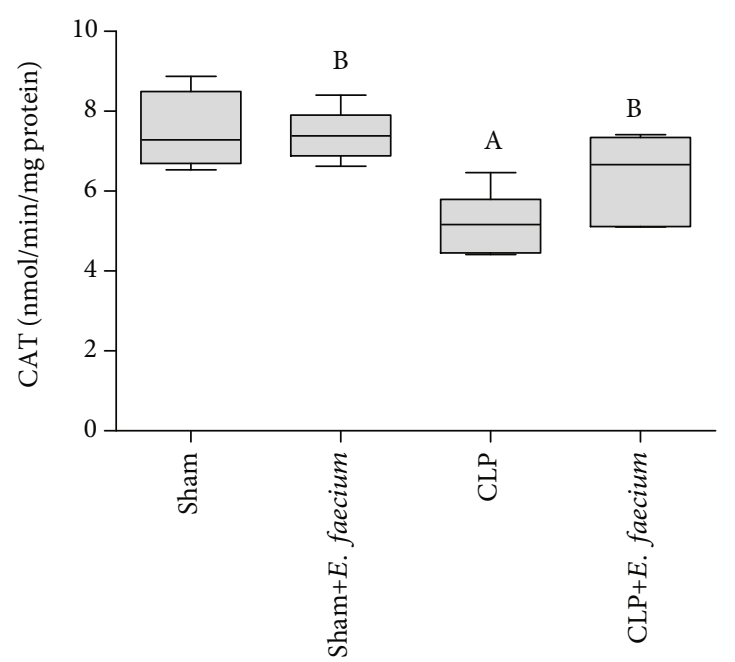

(c)

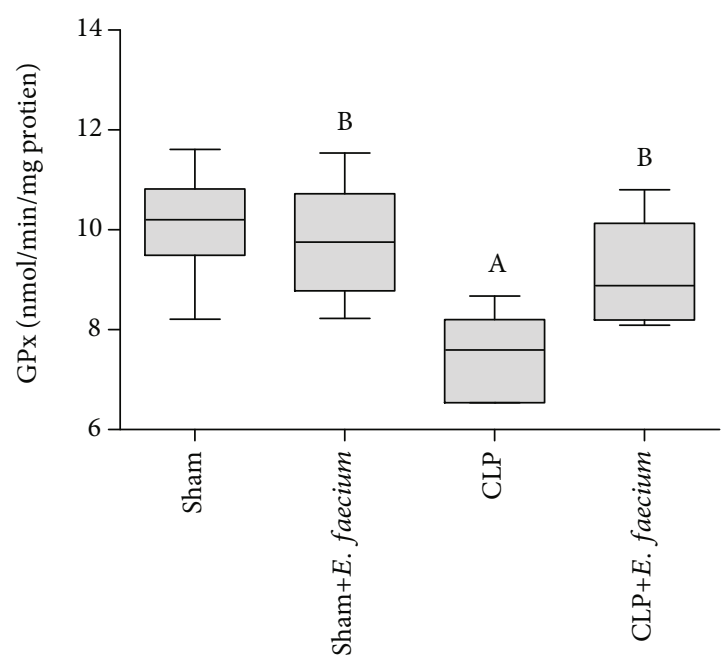

(e)

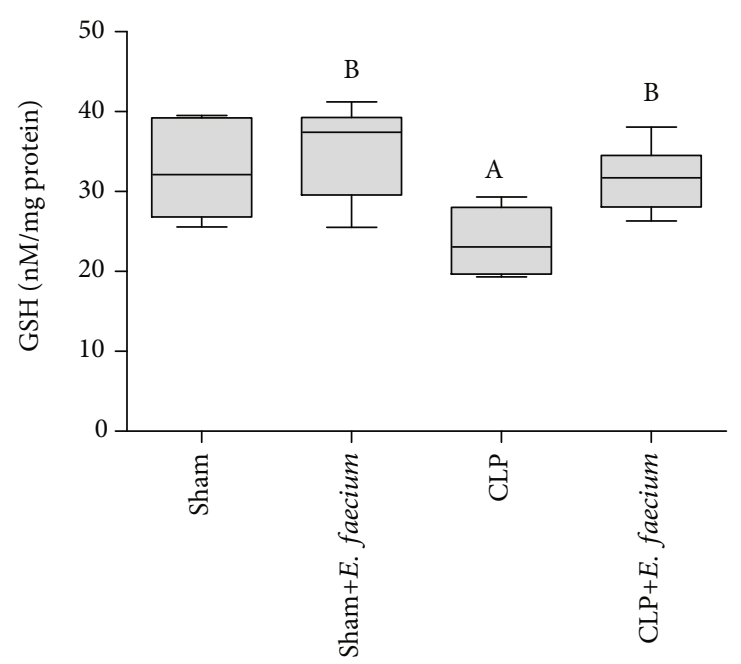

(b)

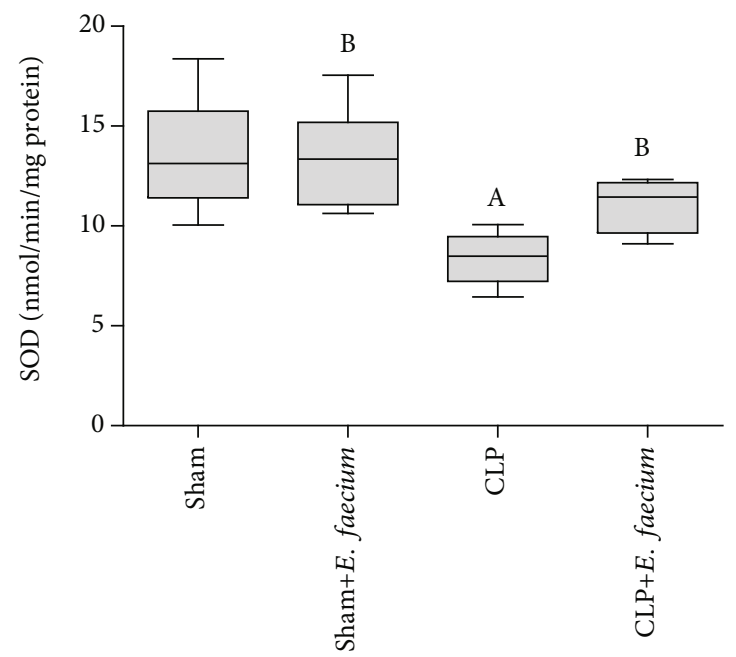

(d)

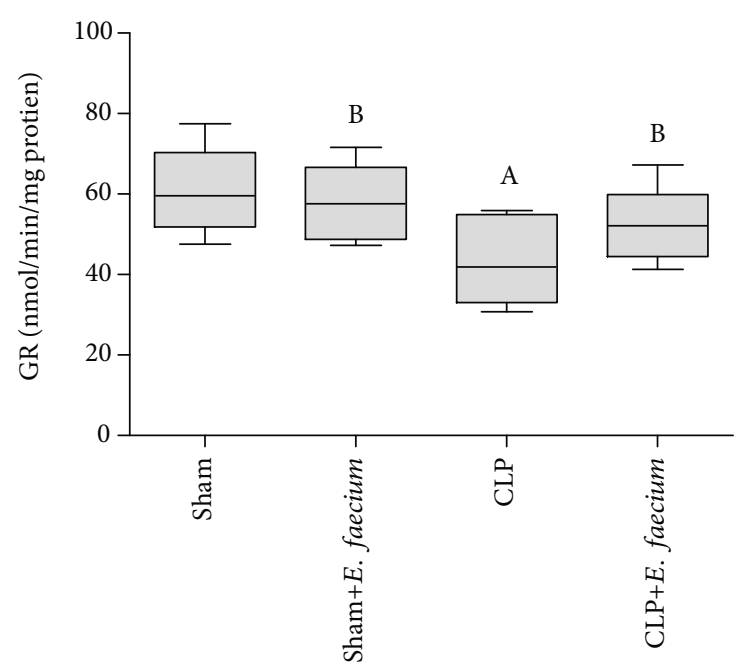

(f)

FIGURE 4: Effects of marine E. faecium pretreatment in Wistar rats with sepsis on lung levels of (a) thiobarbituric acid reaction substances (TBARs), and (b) reduced glutathione (GSH) as well as the enzymatic activities of (c) catalase (CAT), (d) superoxide dismutase (SOD), (e) glutathione peroxidase (GPx), and (f) glutathione reductase (GR). Data are presented as mean \pm SD $(n=6)$, and statistically analyzed by one-way ANOVA followed by the Student-Newman-Keuls post hoc test. Statistical significance was considered when $p \leq 0.05$. Statistical significance was considered when " $A$ " vs. sham group and "B" vs. CLP group. 
TABLE 1: Correlation analysis of inflammatory and oxidative stress biomarkers with lung injury score.

\begin{tabular}{lccccccccc}
\hline & TNF- $\alpha$ & IL-6 & IL-1 $\beta$ & TBARS & GSH & CAT & SOD & GPx & GR \\
\hline Pearson $r$ & 0.3612 & 0.5570 & 0.4377 & 0.4967 & -0.3450 & -0.6660 & -0.4343 & -0.3497 & -0.2313 \\
$p$ value (two-tailed) & 0.0829 & 0.0047 & 0.0324 & 0.0135 & 0.0987 & 0.0004 & 0.0339 & 0.0939 & 0.2768 \\
$R$ squared & 0.1305 & 0.3103 & 0.1916 & 0.2467 & 0.1191 & 0.4436 & 0.1887 & 0.1223 & 0.05350 \\
\hline
\end{tabular}

TABLE 2: Correlation analysis of inflammatory and oxidative stress biomarkers with pulmonary edema.

\begin{tabular}{lccccccccc}
\hline & TNF- $\alpha$ & IL-6 & IL-1 $\beta$ & TBARS & GSH & CAT & SOD & GPx & GR \\
\hline Pearson $r$ & 0.4603 & 0.4588 & 0.4854 & 0.6362 & -0.2630 & -0.3987 & -0.5134 & -0.4469 & -0.4509 \\
$p$ value (two-tailed) & 0.0236 & 0.0241 & 0.0162 & 0.0008 & 0.2143 & 0.0536 & 0.0103 & 0.0286 & 0.0270 \\
$R$ squared & 0.2119 & 0.2105 & 0.2356 & 0.4047 & 0.06917 & 0.1589 & 0.2636 & 0.1997 & 0.2033 \\
\hline
\end{tabular}

significantly lowered in sham animals and CLP animals who had pretreatment with E. faecium $(p \leq 0.05)$ as compared with the CLP group (Figure 2(b)).

Lung tissues of the sham group had significantly lower values of TNF- $\alpha$, IL- 6 , and IL- $1 \beta$ ( $p \leq 0.01,0.001$, and 0.01 , respectively) as compared to the CLP group. These inflammatory mediators were low in the sham $+E$. faecium group ( $p \leq 0.05,0.001$, and 0.05 , respectively) when compared to the CLP group. Pretreatment of CLP animals with E. faecium significantly reduced the lung levels of TNF- $\alpha$, IL-6, and IL- $1 \beta(p \leq 0.05)$ as compared to CLP animals without E. faecium pretreatment (Figure 3 ).

TBARS was assessed in the lung tissues as a marker for lipid peroxidation. TBARS levels in the lung tissues of the CLP group were significantly $(p \leq 0.01)$ higher than those in the sham group. Though, the lung levels of TBARS were significantly low in the sham and CLP groups with $E$. faecium pretreatment ( $p \leq 0.01$ and $p \leq 0.05$, respectively) as compared to the CLP group. The endogenous antioxidant, GSH, was significantly $(p \leq 0.05)$ lower in the CLP group as compared with the sham group. Lung levels of GSH were higher in sham $+E$. faecium and CLP+E. faecium groups ( $p \leq 0.01$ and $p \leq 0.05$, respectively) when compared to the CLP group (Figure 4). The enzymatic activities of antioxidant enzymes, including CAT, SOD, GPx, and GR, were significantly $(p \leq 0.001, p \leq 0.01, p \leq 0.01$, and $p \leq 0.05$, respectively) higher in the sham group as compared to the CLP group. Likewise, CAT, SOD, GPx, and GR activities were significantly $(p \leq 0.001, p \leq 0.01, p \leq 0.01$, and $p \leq 0.05$, respectively) higher in the sham $+E$. faecium group as compared to CLP group. Pretreatment of animals with CLP surgeries with $E$. faecium significantly $(p \leq 0.05)$ increased the enzymatic activities of CAT, SOD, and GPx (Figure 4).

Correlation analysis revealed a significant positive correlation between lung injury score and inflammatory cytokines, including IL-6 and IL-1 $\beta \quad(p=0.0047$ and 0.0324 , respectively). Lung injury score was negatively correlated with TBARS levels $(p=0.0135)$ and the enzymatic activities of CAT and SOD $(p=0.0004$ and 0.0339 , respectively) (Table 1 ). In addition, there was a marked positive correlation between the pulmonary edema and all inflammatory cytokines, including TNF- $\alpha$, IL-6, and IL-1 $\beta$ ( $p=0.0236,0.0241$, and 0.0162 , respec- tively). The lung level of TBARS and the enzymatic activities of SOD, GPx, and GR were also correlated with pulmonary edema significantly $(p=0.0008,0.0103,0.0286$, and 0.0270 , respectively) (Table 2 ).

\section{Discussion}

Pulmonary dysfunction and acute respiratory distress are vital inflammatory complications in patients with sepsis, which result in an increased risk of mortality. Multiple pathogenic microorganisms have been involved in this critical condition. Accordingly, several therapeutic approaches have been introduced to regulate this pathological illness. One major objective is to treat pulmonary-associated inflammation and injury without resistance. In this context, probiotics have been evaluated clinically and experimentally. The prospective therapeutic effects of marine-derived Enterococcus faecium EA9 were evaluated in the present study against sepsis-induced lung inflammation and dysfunction. This study revealed that the probiotics obtained from the marine environment could restore pulmonary injury and edema after CLP surgery. In addition, the elevated markers of inflammation and oxidative stress were markedly corrected to their normal levels in the lungs of CLP animals following the pretreatment with Enterococcus faecium EA9.

The CLP model of sepsis has been widely used in rodents. Experimental evidence suggests the successes of this model. Pathogens might migrate from the intervention site and cause multiple internal organ infections, injuries, and dysregulations. This model showed marked alteration in pulmonary functions and induced marked inflammatory pathways. In Chen et al.'s [21] study, CLP remarkably injured young rats' lungs causing edema and apoptosis. Another experimental study conducted on Wistar rats using the same animal model showed that dexmedetomidine, a well-known anti-inflammatory, restored lung inflammation, edema, and apoptosis [22]. In the present study, CLP induced significant pulmonary injury and altered the normal histological features. The hyperinflammatory status in the lungs of CLP-operated animals that was characterized by a significant increase in inflammatory cytokines was also reported in other studies [23]. Moreover, the CLP model caused a significant pulmonary edema as indicated by 
the increased wet/dry ratio. Previous studies also documented comparable pulmonary edema using the same animal model $[19,24]$.

Reactive oxygen metabolites are considered crucial caustic mediators in the pathogenesis of lung injuries during sepsis [25]. Accordingly, the sepsis model in experimental animals may trigger pulmonary oxidative stress by limiting the endogenous antioxidant (GSH) levels and restricting the activities of antioxidant enzymes. These effects were reported in the present study along with marked lipid peroxidation. The elevated lung levels of TBARS in CLP animals indicate exaggerated cellular membrane damage and apoptosis due to the accumulation of ROS, which affects cellular fluidity and permeability resulting in dysfunction. SOD and CAT are essential antioxidant enzymes that scavenge ROS such as hydrogen peroxide and convert them into oxygen and water. Other antioxidant protective enzymes such as GPx and GR decrease the levels of lipid hydroperoxide and hydrogen peroxide. The CLP model restricts the antioxidant enzyme activities and triggers inflammatory cytokines in vital organs such as the kidney and heart [26]. Notably, studies have suggested that oxidative stress may play a vital role in the pathogenesis of sepsis-induced lung injury and edema. In Emel and Hilal's [27] study, CLP triggered pulmonary oxidative stress and lipid peroxidation along with a reduction in the activities of antioxidant enzymes, including the myeloperoxidase (MPO), SOD, and CAT. Furthermore, another study showed the association between acute lung injury in septic rats and cellular oxidative damages [28].

The health benefits of probiotics are documented, and accordingly, several biomedical applications were introduced [29]. For instance, probiotics might enhance the health conditions and meat production capabilities of broilers and goats [30, 31]. Probiotics also improve the sustainability and performance of marine animals [32]. The probiotic use to manage multiple organs' inflammatory response and cellular damage in the CLP model of sepsis has been introduced in several experimental studies. In one study, mice with sepsis-induced encephalopathy were treated with the probiotic Clostridium butyricum. This treatment alleviated the cognitive impairments and exerted neuroprotective effects [33]. Lactobacillus rhamnosus GG, as a probiotic, was able to reduce CLP-associated liver damages, hypoxic hepatitis, and inflammation in septic rats [34], while, in CLP septic C57BL6 mice, Lactobacillus rhamnosus GG enhanced intestinal permeability and ileum mucosal damage $[35,36]$. Guo et al. reported that pretreatment with a mixture of Bacillus subtilis and Enterococcus faecium can reveal significant intestinal protective effects in septic mice via counteracting intestinal inflammation using the CLP model [37]. Interestingly and similar to our study, treatment of FVB/N mice with either Lactobacillus rhamnosus GG or Bifidobacterium longum markedly lowered lung inflammatory neutrophilic infiltrates following sepsis induction by the CLP model in Khailova et al.'s study [14]. Our findings are in agreement with the trend that probiotic treatment may represent a safe and less resistant approach in sepsis. The aquatic probiotic used in the present study reduced pulmonary hyperinflammation and restored lung injury, histological features, and edema.
Marine microorganisms have a wide taxonomic diversity. They live in a unique habitat subjected to extreme environmental conditions, which necessitate the development of different adaptation mechanisms to withstand these unusual surroundings. They develop a variety of novel secondary metabolites and bioactive compounds with different prospective and structures that cannot be obtained from terrestrial microorganisms [17]. Several attempts were made to produce probiotics from the marine environment. Different marine probiotic bacteria, which belong to phylum Proteobacteria such as Pseudomonas, Shewanella, and Phaeobacter, had an excellent impact on the growth and immune response of fish cultures [38]. One study showed that a marine isolate Psychrobacter maritimus S could enhance the growth and performance of Nile tilapia fingerlings fish [39]. Another study demonstrated that the marine probiotic, Bacillus pumilus $\mathrm{H} 2$, could provide marked preventative effects against fish vibriosis [40]. Aquatic probiotic strain, Bacillus velezensis V4, also was able to regulate the growth and development of multiple furuncles in aquaculture creatures [41]. Moreover, marine Lactobacillus plantarum AH 78 improved the immunity and performance of Nile tilapia through regulation of hepatic cytokine gene expressions, including interleukin-4, interleukin-12, and interferon- $\gamma$ [42]. Likewise, our results demonstrated, for the first time, that marine probiotics, including Enterococcus faecium EA9, inhibit sepsis-induced pulmonary dysregulation and inflammation in CLP rats. In addition, the mechanistic pathways responsible for the reported protective effects were evaluated. Enterococcus faecium EA9 reduced oxidative stress and activated the antioxidant enzymatic activities. This could be through free radical scavenging possibilities. Lipid peroxidation was also suppressed in septic rats with Enterococcus faecium EA9 pretreatment suggesting reduced apoptosis and cellular damage as documented in the histological analysis. The ability of Enterococcus faecium EA9 to decrease the pulmonary inflammatory cytokines in CLP animals resulted in a marked reduction of pulmonary edema and enhanced architecture.

In conclusion, the marine isolate Enterococcus faecium EA9 showed antioxidant and anti-inflammatory properties and reduced lipid peroxidation in lung tissues of septic rats who underwent CLP surgeries. These beneficial properties were correlated with the restoration of pulmonary edema and lung injury. This study demonstrated clear evidence that probiotics developed from marine bioresources could represent a beneficial approach for inflammatory conditions, including sepsis.

\section{Data Availability}

The datasets generated and/or analyzed in the present study are included in the manuscript.

\section{Conflicts of Interest}

The authors declare no competing interests regarding the publication of this paper. 


\section{Authors' Contributions}

Hatem Abuohashish performed the conceptualization, investigation, and formal analysis and wrote and prepared the original draft. Eman Zaghloul performed the conceptualization, investigation, and methodology and wrote, reviewed, and edited the manuscript. Amany El Sharkawy performed the investigation, methodology, and visualization. Eman M. Abbas contributed to the investigation and resources. Mohammed M. Ahmed performed the investigation and methodology. Salim S. Al-Rejaie helped in funding acquisition and formal analysis.

\section{Acknowledgments}

The authors express their appreciation to the Deanship of Scientific Research at King Saud University for funding the work through Research Group Project No. RGP-179.

\section{References}

[1] S. L. Lukacs, K. C. Schoendorf, and A. Schuchat, "Trends in sepsis-related neonatal mortality in the United States, 19851998," The Pediatric Infectious Disease Journal, vol. 23, no. 7, pp. 599-603, 2004.

[2] J. L. Wynn, P. O. Scumpia, M. J. Delano et al., "Increased mortality and altered immunity in neonatal sepsis produced by generalized peritonitis," Shock, vol. 28, no. 6, pp. 675-683, 2007.

[3] H. R. Flori, D. V. Glidden, G. W. Rutherford, and M. A. Matthay, "Pediatric acute lung injury: prospective evaluation of risk factors associated with mortality," American Journal of Respiratory and Critical Care Medicine, vol. 171, no. 9, pp. 995-1001, 2005.

[4] P. Dahlem, W. M. van Aalderen, and A. P. Bos, "Pediatric acute lung injury," Paediatric Respiratory Reviews, vol. 8, no. 4, pp. 348-362, 2007.

[5] S. E. Erickson, G. S. Martin, J. L. Davis, M. A. Matthay, and M. D. Eisner, "Recent trends in acute lung injury mortality: 1996-2005," Critical Care Medicine, vol. 37, no. 5, pp. 15741579, 2009.

[6] M. Rojas, C. R. Woods, A. L. Mora, J. Xu, and K. L. Brigham, "Endotoxin-induced lung injury in mice: structural, functional, and biochemical responses," American Journal of Physiology. Lung Cellular and Molecular Physiology, vol. 288, no. 2, pp. L333-L341, 2005.

[7] L. E. Morrow, M. H. Kollef, and T. B. Casale, "Probiotic prophylaxis of ventilator-associated pneumonia: a blinded, randomized, controlled trial," American Journal of Respiratory and Critical Care Medicine, vol. 182, no. 8, pp. 1058-1064, 2010.

[8] I. I. Siempos, T. K. Ntaidou, and M. E. Falagas, "Impact of the administration of probiotics on the incidence of ventilator-associated pneumonia: a meta-analysis of randomized controlled trials," Critical Care Medicine, vol. 38, no. 3, pp. 954-962, 2010.

[9] E. Pitsouni, V. Alexiou, V. Saridakis, G. Peppas, and M. E. Falagas, "Does the use of probiotics/synbiotics prevent postoperative infections in patients undergoing abdominal surgery? A meta-analysis of randomized controlled trials," European
Journal of Clinical Pharmacology, vol. 65, no. 6, pp. 561-570, 2009.

[10] A. Aceti, L. Maggio, I. Beghetti et al., "Probiotics prevent lateonset sepsis in human milk-fed, very low birth weight preterm infants: systematic review and meta-analysis," Nutrients, vol. 9, no. 8, p. 904, 2017.

[11] S. K. Angurana, A. Bansal, S. Singhi et al., "Evaluation of effect of probiotics on cytokine levels in critically ill children with severe Sepsis," Critical Care Medicine, vol. 46, no. 10, pp. 1656-1664, 2018.

[12] M. Yilmaz and A. O. Erdem, "The protective role of probiotics in sepsis-induced rats," Ulusal Travma ve Acil Cerrahi Dergisi, vol. 26, no. 6, pp. 843-846, 2020.

[13] M. A. Muftuoglu, S. Isikgor, S. Tosun, and A. Saglam, "Effects of probiotics on the severity of experimental acute pancreatitis," European Journal of Clinical Nutrition, vol. 60, no. 4, pp. 464-468, 2006.

[14] L. Khailova, B. Petrie, C. H. Baird, J. A. Dominguez Rieg, and P. E. Wischmeyer, "Lactobacillus rhamnosus GG and Bifidobacterium longum attenuate lung injury and inflammatory response in experimental sepsis," PLoS One, vol. 9, no. 5, article e97861, 2014.

[15] B. Arribas, M. E. Rodríguez-Cabezas, M. Comalada et al., "Evaluation of the preventative effects exerted by Lactobacillus fermentum in an experimental model of septic shock induced in mice," The British Journal of Nutrition, vol. 101, no. 1, pp. 51-58, 2009.

[16] S. K. Kim, I. Bhatnagar, and K. H. Kang, "Development of marine probiotics: prospects and approach," Advances in Food and Nutrition Research, vol. 65, pp. 353-362, 2012.

[17] P. Honkanen, "Consumer acceptance of (marine) functional food," in Wageningen, pp. 141-154, Wageningen Academic Publishers, 2009.

[18] T. Hongpattarakere, N. Cherntong, S. Wichienchot, S. Kolida, and R. A. Rastall, "_In vitro_prebiotic evaluation of exopolysaccharides produced by marine isolated lactic acid bacteria," Carbohydrate Polymers, vol. 87, no. 1, pp. 846-852, 2012.

[19] M. E. Zubrow, S. S. Margulies, and N. Yehya, "Nordihydroguaiaretic acid reduces secondary organ injury in septic rats after cecal ligation and puncture," PLoS One, vol. 15, no. 8, article e0237613, 2020.

[20] G. Matute-Bello, G. Downey, B. B. Moore et al., “An official American Thoracic Society workshop report: features and measurements of experimental acute lung injury in animals," American Journal of Respiratory Cell and Molecular Biology, vol. 44, no. 5, pp. 725-738, 2011.

[21] G. Chen, Y. Hou, X. Li, R. Pan, and D. Zhao, "Sepsis-induced acute lung injury in young rats is relieved by calycosin through inactivating the HMGB1/MyD88/NF- $\kappa$ B pathway and NLRP3 inflammasome," International Immunopharmacology, vol. 96, article 107623, 2021

[22] G. Karabulut, N. Bedirli, N. Akyürek, and E. Bağrıç̧ı, "Doserelated effects of dexmedetomidine on sepsis-initiated lung injury in rats," Brazilian Journal of Anesthesiology, vol. 71, no. 3, pp. 271-277, 2021.

[23] I. Cinar, B. Sirin, P. Aydin et al., "Ameliorative effect of gossypin against acute lung injury in experimental sepsis model of rats," Life Sciences, vol. 221, pp. 327-334, 2019.

[24] J. T. Yin, B. Wan, D. D. Liu et al., "Emodin alleviates lung injury in rats with sepsis," The Journal of Surgical Research, vol. 202, no. 2, pp. 308-314, 2016. 
[25] P. K. Gonzalez, J. Zhuang, S. R. Doctrow et al., "EUK-8, a synthetic superoxide dismutase and catalase mimetic, ameliorates acute lung injury in endotoxemic swine," The Journal of Pharmacology and Experimental Therapeutics, vol. 275, no. 2, pp. 798-806, 1995.

[26] M. A. Dkhil, R. B. Kassab, S. Al-Quraishy, M. M. Abdel-Daim, R. Zrieq, and A. E. Abdel Moneim, “_Ziziphus spina-christi_ (L.) leaf extract alleviates myocardial and renal dysfunction associated with sepsis in mice," Biomedicine \& Pharmacotherapy, vol. 102, pp. 64-75, 2018.

[27] A. Emel and Y. Hilal, "Antioxidant and ntiinflammatory efficacy of curcumin on lung tissue in rats with sepsis," Journal of Traditional Chinese Medicine, vol. 40, no. 5, pp. 820-826, 2020.

[28] W. Xia, Z. Pan, H. Zhang, Q. Zhou, and Y. Liu, "Inhibition of $\operatorname{ERR} \alpha$ aggravates sepsis-induced acute lung injury in rats via provoking inflammation and oxidative stress," Oxidative Medicine and Cellular Longevity, vol. 2020, Article ID 2048632, 9 pages, 2020.

[29] S. K. Korada, N. S. Yarla, V. Mishra, M. A. Daim, B. Sharma, and A. Gm, "Single probiotic versus multiple probiotics - a debate on current scenario for alleviating health benefits," Current Pharmaceutical Design, vol. 24, no. 35, pp. 4150-4153, 2019.

[30] I. F. Rehan, M. Youssef, M. A. M. Abdel-Rahman et al., "The impact of probiotics and egg yolk IgY on behavior and blood parameters in a broiler immune stress model," Front Vet Sci, vol. 7, p. 145, 2020.

[31] A. S. Mandour, H. Samir, M. A. El-Beltagy et al., "Effect of supra-nutritional selenium-enriched probiotics on hematobiochemical, hormonal, and Doppler hemodynamic changes in male goats," Environmental Science and Pollution Research International, vol. 27, no. 16, pp. 19447-19460, 2020.

[32] M. A. O. Dawood, S. Koshio, M. M. Abdel-Daim, and H. Van Doan, "Probiotic application for sustainable aquaculture," Reviews in Aquaculture, vol. 11, no. 3, pp. 907-924, 2019.

[33] J. Liu, Y. Jin, H. Li et al., "Probiotics exert protective effect against sepsis-induced cognitive impairment by reversing gut microbiota abnormalities," Journal of Agricultural and Food Chemistry, vol. 68, no. 50, pp. 14874-14883, 2020.

[34] L. Ding, Y. Gong, Z. Yang et al., "Lactobacillus rhamnosus GG ameliorates liver injury and hypoxic hepatitis in rat model of CLP-induced sepsis," Digestive Diseases and Sciences, vol. 64, no. 10, pp. 2867-2877, 2019.

[35] L. Chen, H. Li, J. Li, Y. Chen, and Y. Yang, "Lactobacillus rhamnosus GG treatment improves intestinal permeability and modulates microbiota dysbiosis in an experimental model of sepsis," International Journal of Molecular Medicine, vol. 43, no. 3, pp. 1139-1148, 2019.

[36] L. Chen, K. Xu, Q. Gui, Y. Chen, D. Chen, and Y. Yang, "Probiotic pre-administration reduces mortality in a mouse model of cecal ligation and puncture-induced sepsis," Experimental and Therapeutic Medicine, vol. 12, no. 3, pp. 1836-1842, 2016.

[37] L. Guo, M. Meng, Y. Wei et al., "Protective effects of live combined B. subtilis and E. faecium in polymicrobial sepsis through modulating activation and transformation of macrophages and mast cells," Frontiers in Pharmacology, vol. 9, p. 1506, 2019.

[38] J. A. Vázquez, A. Durán, M. Nogueira et al., "Production of marine probiotic bacteria in a cost-effective marine media based on peptones obtained from discarded fish by-products," Microorganisms, vol. 8, no. 8, p. 1121, 2020.
[39] S. O. Makled, A. M. Hamdan, and A. F. M. el-Sayed, "Growth promotion and immune stimulation in Nile tilapia, Oreochromis niloticus, fingerlings following dietary administration of a novel marine probiotic, Psychrobacter maritimus S.," Probiotics and Antimicrobial Proteins, vol. 12, no. 2, pp. 365-374, 2020.

[40] X. Y. Gao, Y. Liu, L. L. Miao, E. W. Li, T. T. Hou, and Z. P. Liu, "Mechanism of anti-Vibrio activity of marine probiotic strain Bacillus pumilus $\mathrm{H} 2$, and characterization of the active substance," AMB Express, vol. 7, no. 1, p. 23, 2017.

[41] X. Y. Gao, Y. Liu, L. L. Miao et al., "Characterization and mechanism of anti-Aeromonas salmonicida activity of a marine probiotic strain, Bacillus velezensis V4," Applied Microbiology and Biotechnology, vol. 101, no. 9, pp. 37593768, 2017.

[42] A. M. Hamdan, A. F. M. el-Sayed, and M. M. Mahmoud, "Effects of a novel marine probiotic,Lactobacillus plantarumAH 78, on growth performance and immune response of Nile tilapia (Oreochromis niloticus)," Journal of Applied Microbiology, vol. 120, no. 4, pp. 1061-1073, 2016. 